\title{
Implicación paterna post divorcio: una revisión sistemática
}

Father involvement after divorce: a systematic review

\section{Envolvimento paterno após o divórcio: uma revisão sistemática}

\author{
Lucía Lizarazu ${ }^{1}$, ORCID 0000-0003-0666-429X \\ Cecilia Cracco $^{2}$, ORCID 0000-0003-3953-001X \\ 12 Universidad Católica del Uruguay
}

Resumen: El divorcio o la separación conyugal implica cambios en la estructura de las familias y en los arreglos de convivencia que han estado marcados por sesgos de género. Reconociendo la importancia de la implicación del padre en el desarrollo de los hijos y el factor protector que supone en estos escenarios, se realizó una revisión sistemática de la literatura con el objetivo de conocer las variables que se vinculan a la implicación de padres no residentes. Aplicando criterios de búsqueda y selección específicos, se analizaron 14 artículos de investigación. Los resultados reconocen la incidencia de variables sociodemográficas, de residencia, vinculadas a los arreglos familiares y al tipo de vínculo entre los excónyuges. Se discuten las características de los estudios incluidos y las limitaciones de la revisión realizada y se aportan ideas para la investigación del fenómeno vincular en estudio. Se concluye resaltando la necesidad de que las intervenciones psicosociales y/o decisiones judiciales referidas al divorcio consideren los factores que podrían favorecer la implicación del padre, en beneficio de todos los miembros del sistema familiar.

Palabras clave: implicación paterna; padres no residentes; divorcio; revisión sistemática; coparentalidad.

Abstract: Divorce or conjugal separation involves changes in the structure of families and in living arrangements in which gender differences and biases are notable. Considering the importance of father involvement in the development of children and the length to which it is a protective factor, a systematic review was conducted with the aim of identifying the variables that are associated with father involvement in fathers who do not reside with their children. After considering specific search and selection criteria, 14 primary research papers were analyzed. It was found that there is an incidence of sociodemographic variables, residence, family arrangements and relationship between ex-spouses in father involvement. The characteristics of the studies included as well as the limitations of the review are discussed. Further research areas are analyzed and proposed. It is concluded that psychosocial interventions and judicial decisions around divorce should consider the factors that have been outlined as contributing to father involvement as it could benefit all members of the family system.

Keywords: father involvement; non-resident fathers; divorce; systematic review; coparenting.

$(\mathrm{cc}) \mathrm{BY}$

Esta obra está bajo una licencia de Creative Commons Reconocimiento 4.0 Internacional 
Resumo: O divórcio ou a separação conjugal implicam mudanças na estrutura das famílias e nos arranjos de convivência marcados por preconceitos de gênero. Reconhecendo a importância do envolvimento do pai no desenvolvimento dos filhos e o fator de proteção que se supõe nesses cenários, foi realizada uma revisão sistemática de literatura com o objetivo de conhecer as variáveis que se vinculam ao envolvimento de pais não residentes. Aplicando critérios específicos de busca e seleção, foram analisados 14 artigos de investigação. Os resultados reconhecem a incidência de variáveis sociodemográficas, de residência, vinculadas a arranjos familiares, e de tipo de vínculo entre ex-cônjuges. São discutidas as características dos estudos incluídos e as limitações da revisão realizada e são fornecidas ideias para a investigação do fenômeno de vínculo em estudo. Conclui-se destacando a necessidade de que as intervenções psicossociais e / ou decisões judiciais sobre o divórcio considerem os fatores que poderiam favorecer o envolvimento do pai, em benefício de todos os membros do sistema familiar.

Palavras-chave: envolvimento paterno; pais não residentes; divórcio; revisão sistemática; coparentalidade.

Recibido: 17/12/2020

Aceptado: 20/10/2021

Cómo citar:

Lizarazu, L. \& Cracco, C. (2021). Implicación paterna post divorcio: una revisión sistemática. Ciencias Psicológicas, 15(2), e-2408. doi:

https://doi.org/10.22235/cp.v15i2.2408

Correspondencia: Cecilia Cracco, Universidad Católica del Uruguay, Uruguay.E-mail: ccracco@ucu.edu.uy

El aumento de las separaciones y divorcios ha implicado que un importante número de niños conviva solo con uno de sus progenitores (Amato 2014; Walsh, 2012), generando el interés sobre las características de la relación que se instala con el padre no residente y sus efectos en el desarrollo de los hijos (Dunn, 2004). La configuración de la relación con el padre no conviviente ha sido identificada como uno de los factores mediadores entre el divorcio y el ajuste psicológico de los niños (Amato, 1993).

La custodia de los hijos luego de la separación o el divorcio es un tema que genera polémica y expresa sesgos de género en los países de América Latina y el Caribe (IPPF/WHR \& Promundo, 2017; Zicavo, 2016). Se encuentra aún generalizada la noción de que las madres son las cuidadoras "naturales" o las únicas capaces de asegurar el bienestar de los hijos. También en Uruguay continúa siendo frecuente que las sentencias decreten la tenencia de los hijos a la madre, dejando al padre en régimen de visitas (Perrazza \& Gurgitano, 2017). 
Según los primeros resultados de la Encuesta de Nutrición, Desarrollo Infantil y Salud (Endis; Cabella et al., 2016), $22.5 \%$ de los niños uruguayos entre 0 y 3 años convive solo con su madre (32 \% para hogares en situación de pobreza). De los niños que no conviven con su padre, $31.6 \%$ no tiene ningún contacto con él. En un estudio longitudinal representativo de la infancia uruguaya, Pardo, Martín-García, Castro-Martín y Cabella (2019) encontraron que de 177 casos de separación o divorcio entre 2013 y 2016, solo en 8 casos es el padre el cuidador primario.

Los datos muestran la mayor fragilidad o inestabilidad del vínculo padre-hijo luego de la separación o el divorcio. Sin embargo, existe en Uruguay y en la región una creciente preocupación por asegurar la corresponsabilidad en los cuidados (Scavino \& Batthyány, 2019) y el establecimiento de una coparentalidad positiva luego de la separación o el divorcio (Tay-Karapas, Guzmán-González \& Yárnoz-Yaben, 2020).

Efectivamente, en las últimas décadas ha aumentado la comprensión del papel formativo que juegan los padres en el desarrollo infantil (Finzi-Dottan \& Cohen, 2015; Panter-Brick et al., 2014). Los antecedentes de investigación muestran que los padres contribuyen al desarrollo de sus hijos de manera sustancial (Adamsons \& Johnson, 2013; Lee \& Schoppe-Sullivan, 2017; Miller, Thomas, Waller, Nepomnyaschy \& Emory, 2020; Pruett, Pruett, Cowan \& Cowan, 2017). La "nueva paternidad" implica padres activos, cada vez más involucrados en la crianza, buscando superar el modelo paterno tradicional, donde el padre tenía únicamente el rol de proveedor económico y autoridad ante los hijos (Bach, 2019; Lamb, 2010; Marsiglio \& Roy, 2013).

Un modelo de implicación paterna ampliamente aceptado a nivel académico es el propuesto por Lamb, Pleck, Charnov y Levine (1985), que plantea la implicación paterna en términos de tres componentes: (1) la calidad de la interacción del padre con sus hijos, (2) la disponibilidad del padre para la interacción, y (3) el grado de responsabilidad asumido con los hijos. La interacción supone que el padre comparta tiempo y experiencias de la vida diaria con sus hijos; la accesibilidad refiere a que el padre esté disponible tanto física como psicológicamente para atender a sus hijos; por último, la responsabilidad implica que el padre se comprometa con el bienestar y el cuidado de los niños, haciendo todos los arreglos necesarios para asegurar su subsistencia y cuidado (Lamb et al., 1985).

Los padres expresan dificultad para continuar realizando estas funciones cuando se encuentran separados del otro progenitor (Della Casa \& Käppler, 2009). El contacto con el padre no residente es necesario, pero no suficiente para considerar que se ha establecido una buena implicación; es el tipo de actividades en la que los padres no residentes se involucran y la calidad del tiempo que comparten lo que incide en el bienestar de los hijos (Adamsons \& Johnson, 2013). Los niños afirmaron sentir una relación cercana con el padre cuando él se comprometió emocionalmente con ellos y les mostró su atención más allá de la frecuencia de visitas (Della Casa, Spillner, Winkler-Metzke \& Steinhausen, 2012).

Los padres no residentes son una población difícil de pesquisar (Bucheli \& Vogorito, 2015). El acceso a los padres a menudo se da a través de las madres (Della Casa \& Käppler, 2009) y aunque se los contacte aleatoriamente suelen ser solo los más implicados los que participan de las investigaciones (Adamsons \& Johnson, 2013). Se reconoce además que las madres tienden a sub reportar y los padres a sobre reportar la implicación, especialmente en familias en que los padres no están en pareja (Adamsons \& Johnson, 2013; Charles et al., 2018). 
Reconociendo la importancia de la relación padre-hijos luego del divorcio, Oliveira y Crepaldi (2018) realizaron una revisión de trabajos empíricos publicados entre 2005 y 2016 en las bases de datos PubMed, APA, PsycNET, Web of Science y Scielo Brasil con los términos divorcio y relación padre-hijos, tanto en inglés como en portugués. Las autoras seleccionaron 25 trabajos ( 21 en inglés y 4 en portugués; 22 cuantitativos y 3 cualitativos) cuyo análisis les permitió identificar factores que favorecen la relación con el padre y otros que la obstaculizan, destacando los efectos de la presencia o no de esa relación en el desarrollo de los hijos.

Este trabajo busca precisar, ampliar y actualizar la revisión realizada por Oliveira y Crepaldi (2018). Se realizará un estudio con criterios básicos de las revisiones sistemáticas para identificar las variables que se vinculan a la implicación paterna post-divorcio o separación conyugal. En concordancia con el marco conceptual desarrollado, en esta revisión se buscaron investigaciones que refieran específicamente a la implicación paterna (en lugar de relación padre-hijos), seleccionado únicamente los trabajos de tipo cuantitativo para simplificar el análisis conjunto de los resultados. Respecto al trabajo antes citado, esta revisión amplía las bases de datos consultadas e incluye trabajos publicados en inglés y en español hasta el año 2020.

\section{Método}

\section{Estrategias de búsqueda y criterios de elegibilidad de los artículos}

En la tabla 1 se presentan las bases de datos consultadas, descriptores utilizados y criterios establecidos para la búsqueda documental.

Tabla 1.

Criterios para la búsqueda documental de artículos

Bases de datos consultadas Academic Search Complete, Fuente Académica Plus, Fuente Académica Premier, Medline, SocINDEX with Full Text, Psychology and Behavioral Sciences Collection, PsycARTICLES, SienceDirect, Supplemental Index, Complementary Index

Descriptores básicos "Implicación paterna" "Father involvement" en resumen AND "padres no residentes" "non resident fathers" OR "non-resident fathers" en todos los campos

Criterios de inclusión

Estudios cuantitativos en los que la muestra incluya y distinga padres no residentes, y que la custodia de los niños la tenga su madre

Otros filtros

Texto completo en vínculo

Publicaciones arbitradas

Idiomas: inglés y español 


\section{Procedimiento}

Fase 1. El 16 de mayo de 2020 se consultaron las bases de datos siguiendo los criterios presentados en la tabla 1, obteniéndose un total de 88 trabajos.

Fase 2. De los 88 trabajos, el buscador eliminó automáticamente 28 repeticiones, obteniéndose un total de 60 artículos.

Fase 3. De estos 60 artículos se realiza una depuración por título y/o resumen y se eliminan 40 (figura 1).

Fase 4. Se realiza una segunda depuración por texto completo. A los 13 artículos que refieren a factores que favorecen o interfieren con la implicación de padres no residentes, se agrega manualmente un artículo que cumple con los criterios de elegibilidad por aportar información sobre Uruguay, conformando así un total de 14 artículos a ser analizados.

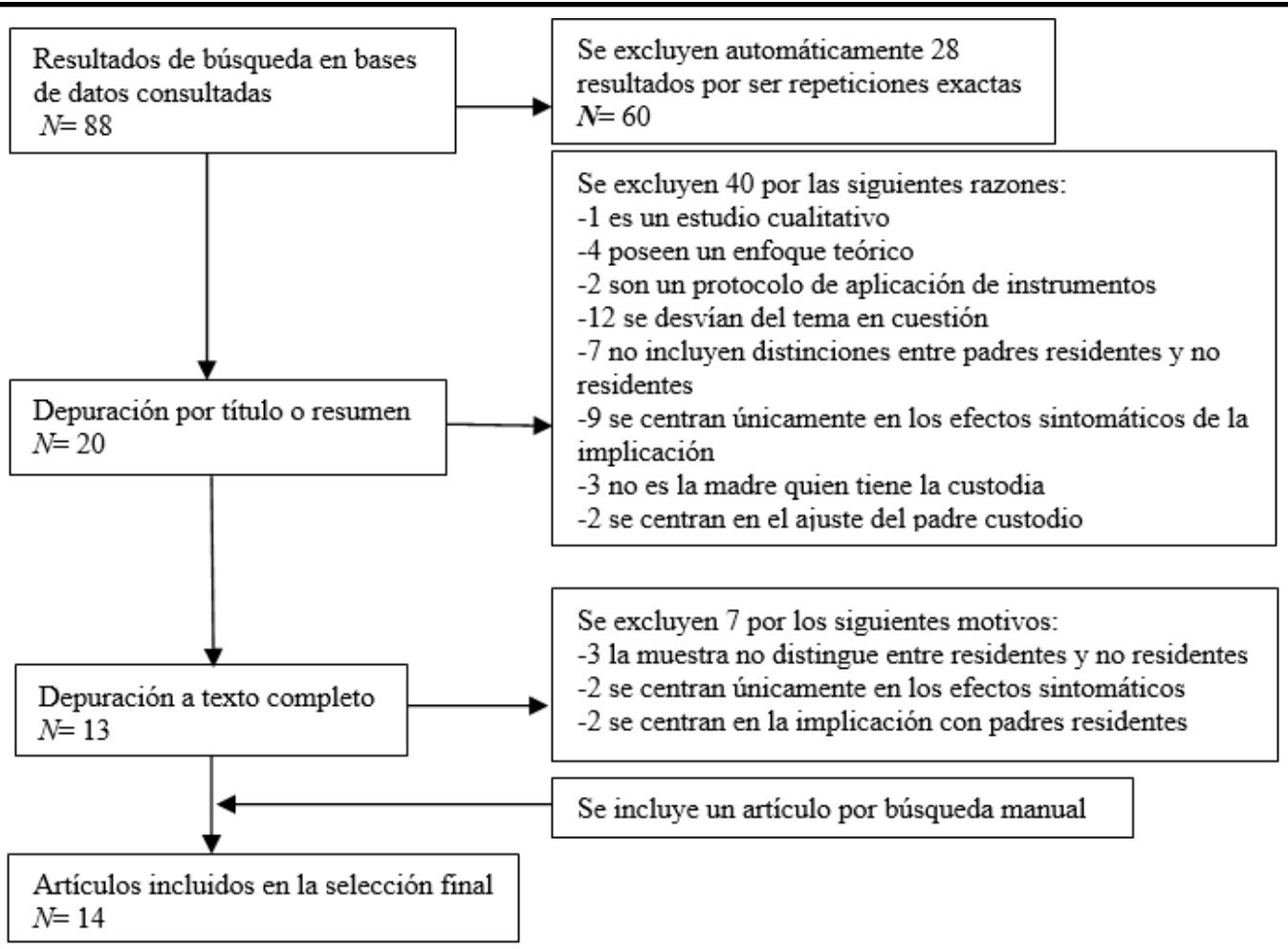

Figura 1. Flujo del proceso realizado para la inclusión y depuración de estudio. 


\section{Resultados}

\section{Presentación de las investigaciones}

En la tabla 2 se presenta información sobre las investigaciones seleccionadas. Solamente uno de estos artículos (Elam, Sandler, Wolchik \& Tein, 2015) forma parte de los resultados de la revisión de Oliveira y Crepaldi (2018). Los trabajos fueron publicados entre 2003 y 2019, siendo la mayoría provenientes de Estados Unidos (Castillo \& Fenzl-Crossman, 2010; Castillo, Welch \& Sarver, 2011; 2012; Elam et al., 2015; Garasky, Stewart, Gundersen \& Lohman, 2010; Jessee \& Adamsons, 2018; Manning, Stewart \& Smock, 2003; Perry, 2009; Tamis-LeMonda, Kahana-Kalman \& Yoshikawa, 2009; Waller, 2012), aunque también se comprenden estudios provenientes de Holanda (Westphal, Poortman \& Van der Lippe, 2014), Jamaica (Devonish \& Anderson, 2017), Sudáfrica (De Wit, Louw \& Louw, 2014) y Uruguay (Pardo et al., 2019).

En los artículos analizados quien aportó los datos acerca de la implicación fue la madre residente (Elam et al., 2015; Garasky et al., 2010; Pardo et al., 2019; Tamis-LeMonda et al., 2009; Waller, 2012), el propio padre (Castillo et al., 2011; 2012; Castillo \& FenzlCrossman, 2010; Devonish \& Anderson, 2017; Manning et al., 2003; Perry, 2009), ambos padres (Jessee \& Adamsons, 2018), madre o padre indistintamente (Westphal et al., 2014), o el hijo en común (Elam et al., 2015; De Wit et al., 2014; Garasky et al., 2010; Jessee \& Adamsons, 2018).

Tabla 2.

Resultados de la revisión

\begin{tabular}{|c|c|c|c|c|}
\hline Autores & Objetivo & Diseño & Muestra & Instrumentos \\
\hline $\begin{array}{l}\text { Castillo \& } \\
\text { Fenzl- } \\
\text { Crossman, } \\
(2010)\end{array}$ & $\begin{array}{l}\text { Determinar si las redes } \\
\text { de apoyo (relación con } \\
\text { la madre del hijo no } \\
\text { residente, familia y/o } \\
\text { amigos, programas } \\
\text { gubernamentales, } \\
\text { religiosos, ONG) se } \\
\text { relacionan con la } \\
\text { implicación de padres } \\
\text { no residentes. }\end{array}$ & $\begin{array}{l}\text { Estudio } \\
\text { cuantitativo, } \\
\text { transversal, } \\
\text { descriptivo, } \\
\text { correlacional } \\
\text { de alcance } \\
\text { explicativo. }\end{array}$ & $\begin{array}{l}\text { Datos de Fragile } \\
\text { Families and Child } \\
\text { Well-being Study. } \\
2754 \text { padres no } \\
\text { residentes con hijos } \\
\text { de entre } 0 \text { y } 2 \text { años. }\end{array}$ & $\begin{array}{l}\text { Encuesta sobre redes de } \\
\text { apoyo informales y } \\
\text { formales existentes. } \\
\text { Escala de implicación } \\
\text { paterna y de relación } \\
\text { coparental de Fragile } \\
\text { Families and Child Well- } \\
\text { Being Study. }\end{array}$ \\
\hline $\begin{array}{l}\text { Castillo, } \\
\text { Welch \& } \\
\text { Sarver } \\
(2011)\end{array}$ & $\begin{array}{l}\text { Determinar las } \\
\text { características } \\
\text { (residencia, edad, raza, } \\
\text { etnia, logro educativo e } \\
\text { ingresos) del padre se } \\
\text { vinculan y en qué } \\
\text { medida lo hacen con la } \\
\text { implicación paterna. }\end{array}$ & $\begin{array}{l}\text { Estudio } \\
\text { cuantitativo, } \\
\text { transversal, } \\
\text { correlacional, } \\
\text { de alcance } \\
\text { explicativo. }\end{array}$ & $\begin{array}{l}\text { Muestra } \\
\text { representativa de } \\
\text { Fragile Families and } \\
\text { Child Well-being } \\
\text { Study compuesta por } \\
4898 \text { padres con hijos } \\
\text { de entre } 0 \text { y } 2 \text { años de } \\
\text { edad. }\end{array}$ & $\begin{array}{l}\text { Cuestionarios } \\
\text { sociodemográficos. } \\
\text { Escala implicación } \\
\text { paterna de Fragile } \\
\text { Families and Child Well- } \\
\text { being Study. }\end{array}$ \\
\hline
\end{tabular}




\begin{tabular}{|c|c|c|c|c|}
\hline $\begin{array}{l}\text { Castillo, } \\
\text { Welch \& } \\
\text { Sarver } \\
(2012)\end{array}$ & $\begin{array}{l}\text { Identificar si la } \\
\text { estabilidad laboral y la } \\
\text { flexibilidad en los } \\
\text { horarios de trabajo se } \\
\text { relacionan con el } \\
\text { involucramiento } \\
\text { paterno en padres no } \\
\text { residentes de contexto } \\
\text { vulnerable. }\end{array}$ & $\begin{array}{l}\text { Estudio } \\
\text { cuantitativo, } \\
\text { transversal, } \\
\text { descriptivo, } \\
\text { correlacional } \\
\text { de alcance } \\
\text { explicativo. }\end{array}$ & $\begin{array}{l}\text { Datos de Fragile } \\
\text { Families and Child } \\
\text { Well-being Study: } \\
895 \text { padres no } \\
\text { residentes con hijos } \\
\text { menores a } 1 \text { año y } \\
\text { que informaron } \\
\text { acerca de su situación } \\
\text { financiera. }\end{array}$ & $\begin{array}{l}\text { Escala de implicación } \\
\text { paterna de Fragile } \\
\text { Families and Child Well- } \\
\text { being Study. } \\
\text { Se creó una escala de } \\
\text { flexibilidad laboral. }\end{array}$ \\
\hline $\begin{array}{l}\text { De Wit, } \\
\text { Louw \& } \\
\text { Louw } \\
(2014)\end{array}$ & $\begin{array}{l}\text { Determinar la } \\
\text { cantidad, tipo y } \\
\text { frecuencia del contacto } \\
\text { entre los adolescentes } \\
\text { y sus padres no } \\
\text { residentes, y describir } \\
\text { las características de la } \\
\text { implicación paterna. }\end{array}$ & $\begin{array}{l}\text { Estudio } \\
\text { cuantitativo, } \\
\text { de corte } \\
\text { transversal, } \\
\text { de carácter } \\
\text { exploratorio } \\
\text { con alcance } \\
\text { descriptivo. }\end{array}$ & $\begin{array}{l}\text { Los datos se } \\
\text { recabaron de } 5 \\
\text { escuelas elegidas al } \\
\text { azar. Participaron } 65 \\
\text { adolescentes de } \\
\text { padres separados con } \\
\text { custodia materna. }\end{array}$ & $\begin{array}{l}\text { Se construyó una escala } \\
\text { de contacto e } \\
\text { implicación paterna. }\end{array}$ \\
\hline $\begin{array}{l}\text { Devonish } \\
\& \\
\text { Anderson } \\
(2017)\end{array}$ & $\begin{array}{l}\text { Identificar si la edad, } \\
\text { empleo, situación } \\
\text { residencial, nivel } \\
\text { educativo, estado civil, } \\
\text { comunidad (clase } \\
\text { social) y las creencias } \\
\text { sobre ser un buen } \\
\text { padre inciden en las } \\
\text { acciones de ayuda en } \\
\text { la tarea a sus hijos } \\
\text { fuera del matrimonio. }\end{array}$ & $\begin{array}{l}\text { Estudio } \\
\text { cuantitativo, } \\
\text { transversal, } \\
\text { de carácter } \\
\text { exploratorio, } \\
\text { con alcance } \\
\text { correlacional. }\end{array}$ & $\begin{array}{l}\text { Datos de The Father } \\
\text { Study. Una muestra } \\
\text { de } 252 \text { padres no } \\
\text { residentes se } \\
\text { comparó con } 190 \\
\text { padres de clase } \\
\text { media-baja con hijos } \\
\text { dentro del } \\
\text { matrimonio } \\
\text { únicamente. }\end{array}$ & $\begin{array}{l}\text { Father Nurturer Scale; } \\
\text { Provider \& Family man } \\
\text { subscale. }\end{array}$ \\
\hline $\begin{array}{l}\text { Elam, } \\
\text { Sandler, } \\
\text { Wolchik \& } \\
\text { Tein } \\
(2015)\end{array}$ & $\begin{array}{l}\text { Identificar perfiles de } \\
\text { padres no residentes, } \\
\text { dentro de los } 2 \text { años } \\
\text { posteriores a la } \\
\text { separación a partir de } \\
\text { patrones de } \\
\text { implicación paterna, } \\
\text { apoyo y conflictividad. } \\
\text { Evaluar el ajuste de los } \\
\text { niños } 6 \text { años después. }\end{array}$ & $\begin{array}{l}\text { Estudio } \\
\text { cuantitativo, } \\
\text { longitudinal. }\end{array}$ & $\begin{array}{l}\text { Datos de New } \\
\text { Beginning Program. } \\
\text { Se utilizan los datos } \\
\text { del pretest }(n=240) \text { y } \\
\text { de una entrevista } 6 \\
\text { años posterior a la } \\
\text { intervención ( } n= \\
218) \text {. }\end{array}$ & $\begin{array}{l}\text { Dad Contact/Maternal } \\
\text { Barriers to Father } \\
\text { Contact Scale, } \\
\text { Children's Inventory of } \\
\text { Social Support, } \\
\text { Children's Perception of } \\
\text { Interparental Conflict } \\
\text { Scale, Child behavior } \\
\text { Checklist, Children's } \\
\text { Manifest Anxiety Scale, } \\
\text { y Children's Report of } \\
\text { Parental Behavior } \\
\text { Inventory. }\end{array}$ \\
\hline
\end{tabular}




\begin{tabular}{|c|c|c|c|c|}
\hline $\begin{array}{l}\text { Garasky, } \\
\text { Stewart, } \\
\text { Gundersen } \\
\text { \& Lohman } \\
(2010)\end{array}$ & $\begin{array}{l}\text { Examinar la relación } \\
\text { entre la pensión } \\
\text { alimenticia, apoyo no } \\
\text { monetario y visitas de } \\
\text { los padres no } \\
\text { residentes, según nivel } \\
\text { de ingresos. }\end{array}$ & $\begin{array}{l}\text { Estudio } \\
\text { cuantitativo, } \\
\text { transversal, } \\
\text { con alcance } \\
\text { correlacional. }\end{array}$ & $\begin{array}{l}\text { Datos de la segunda } \\
\text { ola de Child } \\
\text { Development } \\
\text { Supplement (CDS-II) } \\
783 \text { niños de entre } 4 \\
\text { y } 17 \text { años que vivían } \\
\text { con sus madres y } \\
\text { tenían un padre no } \\
\text { residente. }\end{array}$ & $\begin{array}{l}\text { Utilizando los datos del } \\
\text { CDS-II se crearon } \\
\text { escalas de apoyo no } \\
\text { monetario, visitas y } \\
\text { pensión alimenticia. }\end{array}$ \\
\hline $\begin{array}{l}\text { Jessee \& } \\
\text { Adamsons } \\
(2018)\end{array}$ & $\begin{array}{l}\text { Identificar factores que } \\
\text { se asocien con la } \\
\text { calidad de la relación } \\
\text { entre padres e hijos y } \\
\text { si esta calidad puede } \\
\text { ser transmitida de } \\
\text { generación en } \\
\text { generación. }\end{array}$ & $\begin{array}{l}\text { Estudio } \\
\text { cuantitativo, } \\
\text { longitudinal, } \\
\text { correlacional, } \\
\text { de alcance } \\
\text { explicativo. }\end{array}$ & $\begin{array}{l}\text { Datos de Fragile } \\
\text { Families and Child } \\
\text { Well-being Study. } \\
2970 \text { familias cuyos } \\
\text { padre y madre } \\
\text { brindaron } \\
\text { información al } \\
\text { momento del } \\
\text { nacimiento, al año y } \\
\text { a los } 9 \text { años del hijo. }\end{array}$ & $\begin{array}{l}\text { Escalas de relación con } \\
\text { el abuelo paterno, } \\
\text { implicación paterna y } \\
\text { calidad de la relación } \\
\text { entre padre e hijo de } \\
\text { Fragile Families } \\
\text { and Child Well- } \\
\text { being Study. }\end{array}$ \\
\hline $\begin{array}{l}\text { Manning, } \\
\text { Stewart \& } \\
\text { Smock } \\
(2003)\end{array}$ & $\begin{array}{l}\text { Describir las } \\
\text { complejidades de las } \\
\text { configuraciones } \\
\text { familiares de padres no } \\
\text { residentes y evaluar } \\
\text { cómo operan sobre la } \\
\text { implicación paterna } \\
\text { (visitas y pensión } \\
\text { alimenticia). }\end{array}$ & $\begin{array}{l}\text { Estudio } \\
\text { cuantitativo, } \\
\text { transversal, } \\
\text { de alcance } \\
\text { descriptivo y } \\
\text { correlacional. }\end{array}$ & $\begin{array}{l}\text { Datos de la primera } \\
\text { ola del National } \\
\text { Survey of Families } \\
\text { and Households } \\
\text { (NSFH). } 759 \text { padres } \\
\text { no residentes cuyos } \\
\text { hijos vivían con sus } \\
\text { madres biológicas. }\end{array}$ & $\begin{array}{l}\text { Se utilizaron las escalas } \\
\text { del NSFH: frecuencia de } \\
\text { visitas, contribuciones } \\
\text { económicas. Se } \\
\text { construyó una escala } \\
\text { para la complejidad de la } \\
\text { configuración familiar. }\end{array}$ \\
\hline $\begin{array}{l}\text { Pardo, } \\
\text { Martín- } \\
\text { García, } \\
\text { Castro- } \\
\text { Martín \& } \\
\text { Cabella } \\
(2019)\end{array}$ & $\begin{array}{l}\text { Analizar la relación } \\
\text { entre las prácticas } \\
\text { parentales pre } \\
\text { separación y la } \\
\text { implicación paterna } \\
\text { post separación en } \\
\text { Uruguay. }\end{array}$ & $\begin{array}{l}\text { Estudio } \\
\text { cuantitativo, } \\
\text { longitudinal, } \\
\text { de alcance } \\
\text { corelacional. }\end{array}$ & $\begin{array}{l}\text { Datos de la Endis } \\
\text { Uruguay. Madres que } \\
\text { informan sobre } 177 \\
\text { casos de padres } \\
\text { residentes de niños } \\
\text { entre } 0 \text { y } 3 \text { años en } \\
2013 \text { y que en } 2016 \\
\text { reportaron ya no } \\
\text { residir con el padre. }\end{array}$ & $\begin{array}{l}\text { Varias escalas de la } \\
\text { Endis. }\end{array}$ \\
\hline
\end{tabular}




\begin{tabular}{|c|c|c|c|c|}
\hline $\begin{array}{l}\text { Perry } \\
(2009)\end{array}$ & $\begin{array}{l}\text { Examinar en qué } \\
\text { medida las familias } \\
\text { extendidas } \\
\text { proporcionan apoyo al } \\
\text { padre no residente y } \\
\text { cuál es el impacto de } \\
\text { este apoyo en la } \\
\text { implicación paterna en } \\
\text { familias } \\
\text { afroamericanas. }\end{array}$ & $\begin{array}{l}\text { Estudio } \\
\text { cuantitativo, } \\
\text { transversal, } \\
\text { descriptivo, } \\
\text { correlacional, } \\
\text { de alcance } \\
\text { explicativo. }\end{array}$ & $\begin{array}{l}\text { Datos de Fragile } \\
\text { Families and Child } \\
\text { Well-being Study. } \\
278 \text { padres no } \\
\text { residentes de la tercer } \\
\text { ola de entrevistas } \\
\text { (hijos de } 3 \text { años) que } \\
\text { se identificaron a sí } \\
\text { mismos como negros } \\
\text { o afroamericanos. }\end{array}$ & $\begin{array}{l}\text { Parenting Stress } \\
\text { Inventory, Aggravation } \\
\text { in Parenting Scale y } \\
\text { varias escalas de Fragile } \\
\text { Families and Child Well- } \\
\text { Being Study. }\end{array}$ \\
\hline $\begin{array}{l}\text { Tamis- } \\
\text { LeMonda, } \\
\text { Kahana- } \\
\text { Kalman \& } \\
\text { Yoshikawa } \\
(2009)\end{array}$ & $\begin{array}{l}\text { Determinar si existe } \\
\text { variación entre la } \\
\text { implicación prenatal } \\
\text { según la etnia. } \\
\text { Identificar si la etnia, } \\
\text { situación marital y } \\
\text { residencia median } \\
\text { entre la implicación } \\
\text { paterna prenatal y la } \\
\text { implicación a los } 14 \\
\text { meses del hijo. }\end{array}$ & $\begin{array}{l}\text { Estudio } \\
\text { cuantitativo, } \\
\text { de tipo } \\
\text { longitudinal, } \\
\text { con alcance } \\
\text { explicativo. }\end{array}$ & $\begin{array}{l}310 \text { madres de origen } \\
\text { dominicano, } \\
\text { mexicano y } \\
\text { afroamericano fueron } \\
\text { reclutadas poco } \\
\text { después de dar a luz } \\
\text { en tres hospitales de } \\
\text { Nueva York. Luego } \\
\text { se entrevistaron al } \\
\text { mes, a los } 6 \text { meses y } \\
\text { a los } 14 \text { meses ( } n= \\
204) \text {. }\end{array}$ & $\begin{array}{l}\text { Se construyeron escalas } \\
\text { de implicación prenatal, } \\
\text { en la infancia y a los } 14 \\
\text { meses, además una que } \\
\text { evaluaba la relación } \\
\text { entre los progenitores. }\end{array}$ \\
\hline $\begin{array}{l}\text { Waller } \\
(2012)\end{array}$ & $\begin{array}{l}\text { Investigar factores que } \\
\text { predicen el estilo de } \\
\text { coparentalidad. } \\
\text { Examinar cómo estos } \\
\text { estilos (cooperativo, } \\
\text { indiferente, conflictivo } \\
\text { o mixto) se vinculan a } \\
\text { la implicación paterna } \\
\text { y tiempo con el hijo. }\end{array}$ & $\begin{array}{l}\text { Estudio } \\
\text { cuantitativo, } \\
\text { longitudinal, } \\
\text { correlacional, } \\
\text { con alcance } \\
\text { explicativo. }\end{array}$ & $\begin{array}{l}\text { Datos de Fragile } \\
\text { Families and Child } \\
\text { Well-being Study. } \\
2695 \text { casos de padres } \\
\text { y madres no casados } \\
\text { al momento del } \\
\text { nacimiento del hijo } \\
\text { en común. }\end{array}$ & $\begin{array}{l}\text { Escala de cooperación } \\
\text { coparental, conflicto } \\
\text { coparental e implicación } \\
\text { paterna de Fragile } \\
\text { Families and Child Well- } \\
\text { being Study. }\end{array}$ \\
\hline $\begin{array}{l}\text { Westphal, } \\
\text { Poortman, } \\
\& \text { Van der } \\
\text { Lippe } \\
(2014)\end{array}$ & $\begin{array}{l}\text { Describir y explicar los } \\
\text { cambios con el paso } \\
\text { del tiempo en el } \\
\text { contacto diurno y } \\
\text { nocturno entre padres } \\
\text { no residentes y sus } \\
\text { hijos en el primer año } \\
\text { tras la separación. En } \\
\text { diferentes cohortes de } \\
\text { divorcio entre } 1949 \text { y } \\
\text { 1998. }\end{array}$ & $\begin{array}{l}\text { Estudio } \\
\text { cuantitativo, } \\
\text { retrospectivo, } \\
\text { de carácter } \\
\text { descriptivo. }\end{array}$ & $\begin{array}{l}\text { Datos de Netherlands } \\
\text { Survey 1998. } 808 \\
\text { casos que } \\
\text { informaron: el año de } \\
\text { la separación, hijos } \\
\text { menores en común al } \\
\text { momento de la } \\
\text { separación, que } \\
\text { residieran con la } \\
\text { madre y datos } \\
\text { demográficos de la } \\
\text { unidad familiar. }\end{array}$ & $\begin{array}{l}\text { A partir de los datos se } \\
\text { crearon escalas de } \\
\text { contacto diurno y } \\
\text { nocturno con el padre no } \\
\text { residente e implicación } \\
\text { paterna durante el } \\
\text { matrimonio. }\end{array}$ \\
\hline
\end{tabular}




\section{Variables sociodemográficas vinculadas a la implicación paterna}

La residencia fue identificada como un importante predictor del nivel de implicación paterna (Castillo et al., 2011; Tamis-LeMonda et al., 2009). La distancia entre la residencia del niño y del padre no conviviente se asoció negativamente al contacto (Garasky et al., 2010; Westphal et al., 2014) y también a la probabilidad de recibir pensión alimenticia (Garasky et al., 2010; Manning et al., 2003).

No se observaron diferencias significativas en cuanto a la implicación paterna según el género del hijo (De Wit et al., 2014; Tamis-LeMonda et al., 2009). Aunque sí se identifican diferencias en torno a las temáticas y actividades compartidas entre ambos. La edad de los niños se asoció negativamente a las visitas en horario nocturno, siendo más frecuente en niños y menor en adolescentes, ya que pasar la noche en lo del padre no residente puede comprometer actividades sociales (De Wit et al., 2014; Westphal et al., 2014).

Respecto a la edad del padre, los resultados son contradictorios, reportando en algunos casos mayor implicación para padres jóvenes (Castillo \& Fenzl-Crossman, 2010; Castillo et al., 2012; Devonish \& Anderson, 2017; Perry, 2009); mientras que en otros casos la implicación fue mayor para padres de más edad (Castillo et al., 2011; Jessee \& Adamsons, 2018; Manning et al., 2003; Waller, 2012).

Cuando la información es brindada por las madres, estas evalúan más alta la implicación para padres mayores, destacando los componentes de accesibilidad y responsabilidad (correspondientes al modelo de Lamb et al., 1985) por sobre la dimensión de interacción. Las madres pueden sentirse abrumadas, más allá de la parte económica, por tener que tomar decisiones sobre aspectos de la crianza, salud, educación y disciplina, aspectos que aparecen como opcionales para los padres no residentes (De Wit et al., 2014; Della Casa \& Käppler, 2009), quienes eligen compartir las actividades más placenteras con los hijos (Pardo et al., 2019).

Los trabajos de Castillo y Fenzl-Crossman (2010), Castillo et al. (2012), Devonish y Anderson (2017), Jessee y Adamsons (2018), Pardo et al. (2019), Waller (2012) y Westphal et al. (2014) coinciden al señalar que el empleo, nivel educativo e ingreso se vinculan significativa y positivamente a la implicación paterna. El nivel educativo y los recursos económicos se mostraron como factores más influyentes que el grado de implicación preseparación (Pardo et al., 2019). Algunos autores identificaron también que a más alta clase social (Garasky et al., 2010) o autopercepción de la misma aumentan las posibilidades de implicación del padre (Devonish \& Anderson, 2017).

Nivel educativo, empleo, ingreso y clase social son elementos interrelacionados que operan sobre la implicación (Castillo et al., 2012; Devonish \& Anderson, 2017; Jessee \& Adamsons, 2018). El logro educativo está altamente vinculado al tipo de trabajo (formal/informal) (Pardo et al., 2019), y este a los ingresos y a la clase social. Además, los trabajos mejor remunerados suelen ser los que permiten arreglos laborales más flexibles que posibilitan adaptarse a las necesidades familiares (Castillo et al., 2012).

Los padres cuyo nivel educativo les permitió integrarse al mercado laboral pasan más tiempo con sus hijos ayudándolos en la tarea y transmitiendo la importancia de invertir en educación, más allá de compartir o no residencia (Devonish \& Anderson, 2017). La participación del padre en las decisiones educativas del hijo se relaciona más al nivel educativo del padre que a la implicación previa a la separación (Pardo et al., 2019). 
El nivel educativo del padre y su clase social también se asocian positivamente al pago de pensión alimenticia (Pardo et al., 2019). Igualmente, los estudios revisados muestran que las características del hogar en el que el hijo reside son las mejores predictoras para determinar si el hijo recibe o no pensión. Por ejemplo, que el niño viva con otros adultos se asocia negativamente a recibir pensión (Garasky et al., 2010); por el contrario, si hay otros hermanos de la misma unión se vuelve más probable recibir apoyo económico (Manning et al., 2003). Además, Garasky et al. (2010) y De Wit et al. (2014) sostienen que los padres que ven más frecuentemente a sus hijos son más conscientes de sus necesidades económicas, lo que los lleva a aumentar sus contribuciones tanto monetarias como no monetarias (ropa, útiles, pañales).

\section{Variables vinculadas a los arreglos familiares}

Manning et al. (2003) plantean que la literatura sobre padres no residentes comúnmente trata a los padres como actores aislados, ignorando la complejidad de las circunstancias familiares en las que se encuentran. En su estudio, el $51 \%$ de los padres tiene responsabilidades con más de una familia. Los padres en situaciones simples (con solo un conjunto de hijos no residentes) visitan a sus hijos más seguido y tienen un $85 \%$ más de probabilidad de pagar pensión alimenticia que aquellos en situaciones complejas. En esta misma línea, Devonish y Anderson (2017) encontraron que los padres en situaciones simples se sentaron a pensar y a hacer la tarea con ellos más frecuentemente que aquellos con responsabilidades con más de una familia.

Analizando los diferentes tipos de complejidad, se ha constatado que tener hijos de relaciones anteriores se asocia negativamente a la implicación paterna (Manning et al., 2003; Waller, 2012); padres con hijos dentro y fuera del matrimonio reportan menores niveles de implicación con los hijos fuera del matrimonio (Devonish \& Anderson, 2017; Manning et al., 2003); tener más hermanos de una misma unión se asocia positivamente a la implicación del padre no residente (Garasky et al., 2010; Manning et al., 2003; Waller, 2012).

También se destaca la influencia que tienen las nuevas parejas en la implicación con los hijos no residentes, identificando una relación positiva entre el ingreso de la pareja del padre no residente, el pago de pensión y las visitas a sus hijos (Manning et al., 2003; Waller, 2012). Otro factor respecto a la pareja del padre no residente es que, si esta también tiene hijos no residentes, disminuyen las probabilidades de visita del padre (Manning et al., 2003). En el estudio de Devonish y Anderson (2017) uniones más informales entre el padre y su nueva pareja se asocian con mayor implicación, en contraposición a los resultados de Garasky et al. (2010), que señalan que el hecho de que el padre contraiga un nuevo matrimonio se asocia positivamente a las visitas.

La familia extendida también se vincula a la implicación paterna; las redes de apoyo informales (amigos y familia) de los padres están significativa y positivamente asociadas a una mayor implicación (Castillo \& Fenzl-Crossman, 2010). Por el contrario, la relación con la familia extendida materna se asoció negativamente a la implicación en familias afroamericanas; los niños que fueron frecuentemente visitados por su familia extendida materna mostraron padres menos implicados (Perry, 2009). Se desconoce si esta mayor presencia por parte de la familia materna ocurre porque el padre está poco involucrado o si se debe a que queda eclipsado por la presencia de la familia materna aun cuando quiere implicarse. Lo que parece evidente es que la presencia de figuras experimentadas en torno a 
la crianza conduce a una mayor ambigüedad del rol del padre (Perry, 2009). En este sentido, es probable que los padres no residentes sean valorados positivamente por figuras de la familia extendida solo si cumplen primero con el rol de proveedor económico para después dejar espacio a otros planos de la implicación.

Jessee y Adamsons (2018) hallaron que las características de la relación entre padre y el abuelo paterno predicen un $11 \%$ de la variación en la calidad de relación padre e hijo (a la edad de 9 años). Los hombres que experimentaron relaciones positivas con sus padres revivirán más fácilmente comportamientos parentales positivos, ya que estas fueron modeladas (Jessee \& Adamsons, 2018).

\section{Papel de la madre y de los conflictos entre los excónyuges}

Los autores coinciden en que una buena relación con la madre es determinante para una mayor implicación paterna (Castillo \& Fenzl-Crossman, 2010; Perry, 2009; TamisLeMonda et al., 2009). Perry (2009) considera fundamental no solo la relación entre los progenitores, sino también la visión que posee la madre sobre el padre y sus capacidades parentales.

Waller (2012) señala que la historia amorosa entre los padres fue el mayor predictor del estilo de coparentalidad fuera del matrimonio y, a su vez, este estilo influyó decisivamente en la futura implicación paterna. Los padres que se separaron luego del nacimiento tuvieron $9.86 \%$ más probabilidad de establecer un estilo conflictivo, $3.91 \%$ un estilo indiferente y $2.3 \%$ un estilo mixto respecto a los padres que permanecieron en pareja. El estilo de crianza cooperativo se asocia fuertemente a una alta implicación paterna, mientras que los padres de familias con estilos de coparentalidad indiferentes o conflictivos pasan menos tiempo con sus hijos de 36 meses. El estilo conflictivo tiene efectos significativamente más negativos que el estilo indiferente en cuanto a la implicación paterna (Elam et al., 2015; Waller, 2012). Es interesante señalar que el estilo mixto obtuvo resultados similares al cooperativo en términos de implicación, mostrando lo importante que es que los padres logren eventualmente trabajar en equipo (Waller, 2012).

En cuanto a la implicación paterna, coparentalidad y los efectos sobre los hijos, el estudio longitudinal llevado a cabo por Elam et al. (2015) plantea que el bienestar de los niños post divorcio depende de dos factores: el nivel de implicación con el padre no residente y el nivel de conflicto entre los progenitores. El estudio se llevó a cabo con madres divorciadas/separadas hace menos de 2 años, con al menos un hijo bajo su custodia de entre 9 a 12 años, que formaban parte de un programa de intervención para madres e hijos con problemas externalizantes. Evaluando variables de contacto paterno, grado de conflicto interparental y apoyo percibido por el niño, se identificaron 4 perfiles: 1) alto contacto, conflicto moderado, apoyo moderado (16\% de la muestra); los siguientes dos perfiles comparten características de implicación paterna en cuanto a moderado contacto y apoyo, pero difieren en el nivel de conflicto interparental: 2) bajo conflicto, contacto moderado, apoyo moderado (31.8\% de la muestra); 3) alto conflicto, contacto moderado, apoyo moderado ( $24.3 \%$ de la muestra); el cuarto grupo identificado como 4) poco contacto y apoyo y un nivel moderado conflicto ( $27 \%$ de la muestra) corresponde a perfiles de padres que se alejan del rol de la paternidad luego del divorcio (Elam et al., 2015). Ningún grupo se distinguió por el alto nivel de contacto y apoyo paterno, probablemente por las características 
de selectividad de la muestra. En cuanto a los efectos sobre los niños en familias con alto conflicto (grupo 3), puntuaron más alto en trastornos internalizantes y externalizantes que los otros grupos al momento de la intervención. Seis años después, durante la segunda recolección de datos los niños con bajo apoyo y contacto paterno además de conflicto moderado (grupo 4) resultaron tener más trastornos internalizantes y externalizantes que los otros grupos, incluso los de alto conflicto interparental (Elam et al., 2015).

\section{Discusión}

Las investigaciones revisadas en este trabajo presentan características que deben ser consideradas. En primer lugar, la mayoría de los artículos seleccionados proviene de países fuera de la región (la única excepción es el trabajo de Uruguay de Pardo et al., 2019), lo cual es coincidente con lo reportado por Oliveira y Crepaldi (2018), donde solamente 3 de un total de 25 trabajos pertenecen a Sudamérica (Brasil) y de ellos 2 son de tipo cualitativo.

Del total de 14 artículos revisados, 6 utilizaron muestras provenientes del mismo estudio: Fragile Families and Child Wellbeing Study, un estudio longitudinal que siguió a una cohorte de familias de bajos ingresos de los Estados Unidos. La mayoría de los padres de Fragile Families corresponden a población afroamericana, con bajo logro educativo, que fueron reclutados inmediatamente luego del nacimiento de su hijo. Esto implica que los padres que no asistieron a conocer a su bebé no estén contemplados y que las muestras sean más bien homogéneas en cuanto a nivel educativo, empleo y salarios.

En la totalidad de las investigaciones revisadas, la forma más utilizada para medir la implicación paterna fue a partir de cuestionarios, de modo que las respuestas pueden contener cierto grado de subjetividad y deseabilidad social. En esta misma línea, varios estudios indican la poca fiabilidad al recolectar información de una sola fuente, independientemente de si se trata de padres o madres (Della Casa \& Käppler, 2009). Únicamente uno de los trabajos revisados (Jessee \& Adamsons, 2018) investigó sobre implicación paterna consultando a ambos padres. Estas debilidades no son tan fuertes en los resultados de la revisión de Oliveira y Crepaldi (2018), en la que los hijos participaron en 20 estudios, las madres en 12 y los padres en 9, en un total de 25 investigaciones.

Todos los autores complejizan la implicación paterna evaluando más de un factor al conceptualizarla, pero la mayoría recogen casos de niños en los primeros años de vida (0-3), por lo que dimensiones como la accesibilidad y el sentimiento subjetivo de cercanía no se pueden evaluar.

A pesar de las posibles limitaciones que se han señalado, los resultados de esta revisión permiten visualizar la complejidad de los factores asociados a la implicación paterna post divorcio o separación. Los factores sociodemográficos son aquellos sobre los que existe mayor evidencia en los artículos analizados, tal vez por lo sencillo que resulta su evaluación y comparación. Menor distancia entre los hogares de padre e hijos, alto nivel educativo del padre, mejor empleo y mayor ingreso son características que aparecen asociadas positivamente a la implicación paterna post divorcio. Algunos de estos factores también fueron analizados en la revisión de Oliveira y Crepaldi (2018) como aspectos que favorecen la relación padre-hijos post divorcio (residencia, nivel educativo del padre). 
Con la obligatoriedad legal del pago de pensión, la relación entre pago e implicación ha decrecido (Finzi-Dottan \& Cohen, 2015). Aun así, se observa una vinculación entre lo material y la implicancia, dado que los padres implicados tienden a aportar más allá de la pensión, mostrando atención a las necesidades de sus hijos.

Algunas de las variables sociodemográficas mencionadas (nivel educativo del padre, empleo, ingreso) pueden explicar también distintos niveles de implicación paterna en familias biparentales o nucleares. De todas formas, Westphal et al. (2014) y Pardo et al. (2019) identificaron que la implicación pre separación solo explica parcialmente la implicación post separación, lo que llevaría a concluir que son otros los factores importantes para comprender los mayores o menores niveles de implicación de los padres con sus hijos luego del divorcio.

La complejidad de los arreglos familiares tanto del padre no residente como de la madre ha sido señalada como un factor importante (Devonish \& Anderson, 2017; Manning et al., 2003). Los autores hacen referencia a configuraciones familiares de tipo extendida, que podrían tener un rol diferencial según se trata de la madre o del padre no residente. La presencia de otros adultos en el hogar de la madre parece asociarse a menor implicación del padre (Perry, 2009), mientras que la convivencia del padre con su familia de origen parece oficiar como red de sostén para que dicha vinculación se mantenga. Los resultados contradictorios respecto a la incidencia en la implicación paterna de la conformación de familias ensambladas parecen reflejar la complejidad que suele asociarse a este tipo de configuraciones familiares, especialmente en lo relativo a la ambigüedad de los roles (Oliva, Parra, \& Antolín, 2010).

El nivel de conflicto entre los progenitores previo a la separación o divorcio, durante y luego del mismo, es destacado como un factor que interviene en la implicación del padre con sus hijos (Castillo \& Fenzl-Crossman, 2010; Perry, 2009; Tamis-LeMonda et al., 2009) y en el tipo de vínculo de coparentalidad que mantendrán, siendo estos resultados concordantes con lo reportado por Oliveira y Crepaldi (2018).

En la década de los 90, Allen y Hawkins (1999) hablaban de gatekeeping para hacer referencia a cómo las madres, y en menor medida también los propios padres, presentaban cierto grado de ambivalencia en relación con una mayor implicación masculina en el cuidado y la educación de los niños. Schubert, Sesti, Crepaldi y Viera (2018) reflexionan sobre lo paradójico que resulta este fenómeno. Si bien las madres reclaman una participación más activa de los hombres en la crianza de los hijos, parece persistir aún el registro de que esa participación genera interferencias, y hasta cierto desdibujamiento de su rol de madres. En un escenario de mayor conflictividad como el divorcio, estas creencias y comportamientos pueden acentuarse, planteando la necesidad de que los padres aumenten su confianza y convicción en la importancia de mantener y desarrollar el vínculo con sus hijos (Marsiglio \& Roy, 2013).

Las intervenciones psicosociales y los procesos judiciales durante el proceso de divorcio deberían ayudar a disminuir o por lo menos no incrementar el nivel de conflicto entre los excónyuges. Si los sistemas amplios (sistema judicial, educativo, salud, etc.) consideran a los padres como actores relevantes y brindan mensajes claros respecto a la necesidad e importancia de su involucramiento, es posible que los padres se sientan confirmados en el desempeño de su rol. 
El grado de identificación que los hombres posean con el rol paterno y la percepción de lo que pueden aportar para la crianza de su hijo es una construcción compleja, en la que intervienen además aspectos subjetivos. Jessee y Adamsons (2018) señalaron que la historia vincular de los hombres con sus propios padres incide en los comportamientos parentales que los mismos desarrollarán, resultando este aspecto importante de cara a las nuevas generaciones (Schubert et al., 2018).

Tomando en cuenta los resultados de esta revisión y también las limitaciones que fueron señaladas, sería importante continuar investigando la implicación desde los puntos de vista de las madres, los padres y los hijos. Cada uno de los protagonistas otorga importancia a diferentes aspectos de la implicación, por lo que integrando estas miradas se podría capturar mejor la complejidad el fenómeno vincular en estudio. A pesar de las posibles dificultades para contactar a los padres no residentes, sería necesario contar con una descripción más detallada de la visión que tienen los hombres sobre la paternidad (Marsiglio \& Roy, 2013; Schubert et al., 2018). Más investigaciones de corte longitudinal son necesarias para comprender los procesos de adaptación post-divorcio y las variaciones en los niveles de implicación paterna a lo largo de los años y con hijos de diferentes edades.

Ese conocimiento podría aportar información valiosa a los ámbitos judiciales, en donde se toman decisiones que pueden facilitar u obstaculizar la implicación del padre, así como para el diseño de las intervenciones psicosociales que antes, durante y luego de divorcio apoyen a las familias y, en particular, a los padres en el mantenimiento de un vínculo que directa e indirectamente genera efectos beneficiosos sobre el desarrollo y el bienestar de los hijos y de todo el sistema familiar. Estas acciones, asociadas a los cambios en las expectativas culturales y a "la nueva paternidad" podrían colaborar a que un número mayor de padres se implique con sus hijos y desarrolle un mejor lazo de coparentalidad luego de la separación o el divorcio.

\section{Referencias}

Adamsons, K. \& Johnson, S. K. (2013). An updated and expanded meta-analysis of nonresident fathering and child well-being. Journal of Family Psychology, 27(4), 589-599. doi: 10.1037/a0033786

Allen, S. \& Hawkins, A. (1999). Maternal gatekeeping: mothers' beliefs and behaviors that inhibit greater father involvement in family work. Journal of Marriage and the Family, 61, 199-212. doi: 10.2307/353894

Amato, P. \& Gilbreth, J. (1999). Nonresident father and children's well-being: A metaanalysis. Journal of Marriage and the Family, 61, 557-573. doi: 10.2307/353560

Amato, P. (1993). Children's Adjustment to Divorce: Theories, Hypotheses, and Empirical Support. Journal of Marriage and Family, 55(1), 23-38. doi: 10.2307/352954

Amato, P. (2014). The Consequences of Divorce for Adults and Children: An Update. Drustvena Istrazivanja, 23(1), 5-24. doi: 10.5559/di.23.1.01

Bach, A. (2019). The ambiguous construction of nondominant masculinity: configuring the "new" man through narratives of choice, involved fatherhood, and gender equality. Men and Masculinities, 22(2), 338-359. doi: 10.1177/1097184X17715494 
Bucheli, M. \& Vigorito, A. (2015). Después de la ruptura: efectos de la separación en los contactos entre padres e hijos y en el bienestar de las mujeres. En: M. Bucheli, W. Cabella, M. Nathan, P. Fitermann, A. Vigorito \& M. Zerpa, Cambio familiar y bienestar de las mujeres y los niños en Montevideo y el área metropolitana. Una perspectiva longitudinal, (pp. 43-59). Montevideo: Universidad de la República y Unicef Uruguay.

Cabella, W., De Rosa, M., Failache, E. Fitermann, P., Katzkowicz, N., Medina, M. ... \& Vigorito, A. (2016). Salud, nutrición y desarrollo en la primera infancia en Uruguay: primeros resultados de la ENDIS. Recuperado de http://www.ine.gub.uy/c/document_library/get_file?uuid=25fc124a-d876-4040b784-3b25a850b1fa\&groupId=10181

Castillo, J. T. \& Fenzl-Crossman, A. (2010). The relationship between non-marital fathers' social networks and social capital and father involvement. Child \& Family Social Work, 15(1), 66-76. doi: 10.1111/j.1365-2206.2009.00644.

Castillo, J. T., Welch, G. W. \& Sarver, C. M. (2012). Walking a High Beam: The Balance Between Employment Stability, Workplace Flexibility, and Nonresident Father Involvement. American Journal of Men's Health, 6(2), 120-131. doi: $10.1177 / 1557988311417612$

Castillo, J., Welch, G. \& Sarver, C. (2011). Fathering: The Relationship Between Fathers' Residence, Fathers' Sociodemographic Characteristics, and Father Involvement. Maternal \& Child Health Journal, 15(8), 1342-1349. doi: 10.1007/s10995-010-06846

Charles, P., Spielfogel, J., Gorman-Smith, D., Schoeny, M., Henry, D. \& Tolan, P. (2018). Disagreement in parental reports of father involvement. Journal of Family Issues, 39(2) 328-351. doi: 10.1177/0192513X16644639

De Wit, E., Louw, D. \& Louw, A. (2014). Patterns of Contact and Involvement between Adolescents and Their Non-Resident Fathers. Social Work/Maatskaplike Werk, 50(1), 116-133. doi: 10.15270/50-1-19

Della Casa, A. \& Käppler, C. (2009). Das Engagement getrennt lebender Väter Eine Übersicht über den aktuellen Forschungsstand. Praxis Der Kinderpsychologie Und Kinderpsychiatrie, 58(1), 1-15. doi: 10.13109/prkk.2009.58.1.1

Della Casa, A., Spillner, M., Winkler-Metzke, C. \& Steinhausen, H. C. (2012). Familiäre Wahrnehmung und Einflussfaktoren des Engagements getrennt lebender Väter. Praxis der Kinderpsychologie und Kinderpsychiatrie, 61(2), 75-91.

Devonish, J. \& Anderson, P. (2017). Fathering the "Outside" Child: Differences and Shortfalls among Urban Jamaican Fathers. Social \& Economic Studies, 66(1/2), 3377.

Dunn, J. (2004). Annotation: Children's relationships with their nonresident fathers. Journal of Child Psychology and Psychiatry, 45(4), 659-671. doi: 10.1111/j.14697610.2004.00261.x

Elam, K., Sandler, I., Wolchik, S. \& Tein, J.-Y. (2015). Non-Residential Father-Child Involvement, Interparental Conflict and Mental Health of Children Following Divorce: A Person-Focused Approach. Journal of Youth \& Adolescence, 45(3), 581593. doi: 10.1007/s10964-015-0399-5 
Finzi-Dottan, R. \& Cohen, O. (2015). Predictors of Involvement and Warmth of Custodial Fathers in Israel: Comparison with Married and Noncustodial Divorced Fathers. Family Process, 55(1), 171-187. doi: 10.1111/famp.12124

Garasky, S., Stewart, S. D., Gundersen, C. \& Lohman, B. J. (2010). Toward a Fuller Understanding of Nonresident Father Involvement: An Examination of Child Support, In-Kind Support, and Visitation. Population Research \& Policy Review, 29(3), 363-393. doi: 10.1007/s11113-009-9148-3

IPPF/WHR \& Promundo (2017). Estado de la paternidad: América Latina y el Caribe. Nueva York: IPPF/RHO, Washington: Promundo-US.

Jessee, V. \& Adamsons, K. (2018). Father Involvement and Father-Child Relationship Quality: An Intergenerational Perspective. Parenting, 18(1), 28-44. doi: $10.1080 / 15295192.2018 .1405700$

Lamb, M. (2010). The role of the father in child development ( $5^{\circ}$ ed.). New Jersey: John Wiley \& Sons.

Lamb, M., Pleck, J., Charnov, E. \& Levine, J. (1985). Paternal Behavior in Humans. American Zoologist, 25, 883-894.

Lee, J. \& Schoppe-Sullivan, S. (2017). Resident fathers' positive engagement, family poverty, and change in child behavior problems. Family Relations, 66(3), 484-496. doi: 10.1111/fare.12283

Manning, W. D., Stewart, S. D. \& Smock, P. J. (2003). The Complexity of Father's Parenting Responsibilities and Involvement with Nonresident Children. Journal of Family Issues, 24(5), 645-667. doi: 10.1177/0192513x03024005004

Marsiglio, W. \& Roy, K. (2013). Fathers' nurturance of children over the life course. En: G.W. Peterson \& K. R. Bush (eds.), Handbook of Marriage and the Family (pp. 353376). New York: Springer.

Miller, D., Thomas, M., Waller, M., Nepomnyaschy, L. \& Emory, A. (2020). Father Involvement and Socioeconomic Disparities in Child Academic Outcomes. Journal of Marriage and Family, 82(2), 515-533. doi: 10.1111/jomf.12666

Oliva, A., Parra, A. \& Antolín, L. (2010). Familias reconstituidas. En: E. Arranz \& A. Delgado (Coords.), Desarrollo psicológico en las nuevas estructuras familiares (pp. 69-88). Madrid: Pirámide.

Oliveira, J. L. \& Crepaldi, M. A. (2018). Relação entre o pai e os filhos após o divórcio: revisão integrativa da literatura. Actualidades En Psicología, 32(124). doi: 10.15517/ap.v32i124.29021

Panter-Brick, C., Burgess, A., Eggerman, M., McAllister, F., Pruett, K. \& Leckman, J. F. (2014). Practitioner Review: Engaging fathers - recommendations for a game change in parenting interventions based on a systematic review of the global evidence. Journal of Child Psychology and Psychiatry, 55(11), 1187-1212. doi: 10.1111/jcpp.12280

Pardo, I., Martín-García, T., Castro-Martín, T. \& Cabella, W. (2019). Fatherhood after union breakup in Uruguay: transitory or life-long commitment? Journal of Family Issues, 0192513X1988296. doi: 10.1177/0192513x19882960

Perrazza, R. \& Gurgitano, A. (2017). Niñez Judicializada en Uruguay. Montevideo: Psicointegra. 
Perry, A. (2009). The Influence of the Extended Family on the Involvement of Nonresident African American Fathers. Journal of Family Social Work, 12(3), 211-226. doi: 10.1080/10522150903046390

Pruett, M., Pruett, K., Cowan, C. \& Cowan, P. (2017). Enhancing father involvement in lowincome families: a couples group approach to preventive intervention. Child Development, 88(2), 398-407. doi: 10.1111/cdev.12744

Scavino, S. \& Batthyány, K. (2019). Caminos hacia la corresponsabilidad: los varones en el cuidado infantil en Uruguay. Cadernos Pagu, (56), e195621. doi: $10.1590 / 18094449201900560021$

Schubert, M., Sesti, A. P., Crepaldi, M. A. \& Vieira, M. L. (2018). A paternidade e fatores associados ao envolvimento paterno. Nova Perspectiva Sistêmica, 61, 100-119. doi: 10.38034/nps.v27i61.417

Tamis-LeMonda, C., Kahana-Kalman, R. \& Yoshikawa, H. (2009). Father Involvement in Immigrant and Ethnically Diverse Families from the Prenatal Period to the Second Year: Prediction and Mediating Mechanisms. Sex Roles, 60(7-8), 496-509. doi: 10.1007/s11199-009-9593-9

Tay-Karapas, K., Guzmán-González, M. \& Yárnoz-Yaben, S. (2020). Evaluación de la Adaptación al Divorcio-Separación: Propiedades Psicométricas del CAD-S en el Contexto Chileno. Psykhe (Santiago), 29(2), 1-10. doi: 10.7764/psykhe.29.2.1484

Waller, M. R. (2012). Cooperation, Conflict, or Disengagement? Coparenting Styles and Father Involvement in Fragile Families. Family Process, 51(3), 325-342. doi: 10.1111/j.1545-5300.2012.01403.x

Walsh, F. (2012). The new normal. Diversity and complexity in $21^{\text {st }}$-century families. En F. Walsh (Ed.), Normal family processes. Growing diversity and complexity ( $\left.4^{\circ} \mathrm{ed}.\right)$, (pp. 3-27). New York: The Guilford Press.

Westphal, S. K., Poortman, A.-R. \& Van der Lippe, T. (2014). Non-resident Father-Child Contact across Divorce Cohorts: The Role of Father Involvement during Marriage. European Sociological Review, 30(4), 444-456. doi: 10.1093/esr/jcu050

Zicavo, N. (Coord.). (2016). Parentalidad y Divorcio. (Des)encuentros en la familia latinoamericana. ALAFEPSI Editorial.

Contribución de los autores: a) Concepción y diseño del trabajo; b) Adquisición de datos; c) Análisis e interpretación de datos; d) Redacción del manuscrito; e) revisión crítica del manuscrito.

L. L. ha contribuido en a, b, c, d, e; C. C. en c, d, e.

Editora científica responsable: Dra. Lilian R. Daset. 